\title{
Type 2 Diabetes Diagnosis and Treatment Using mHealth
}

\author{
Martin Potančok ${ }^{1, a, *}$, Martin Antoš ${ }^{1, b}$ \\ ${ }^{1}$ Department of Information Technologies, University of Economics, Prague, Prague, Czech Republic \\ amartin.potancok@vse.cz, bantm02@vse.cz \\ *corresponding author
}

Keywords: Diabetes, Digital Health, Healthcare, mHealth, Type 2 Diabetes

\begin{abstract}
The number of patients with diabetes has been rising steadily. Statistics show a worldwide increase from 108 million in 1980 to 422 million in 2014 [1]. Most diabetics in the world suffer from type 2 diabetes. Successful treatment requires greater motivation from patients due to its symptoms. Currently, mHealth solutions are being expanded to support diagnostic and treatment processes. Unfortunately, in many healthcare facilities wider implementation of mHealth and its elements has not yet been successful. The aim of this paper therefore is to specify mHealth implementation into diabetes type 2 diagnostic and treatment processes, including the effects of patient motivation. The research was designed as a qualitative research, namely, a case study. The results indicate that there are benefits for both healthcare facilities and patients.
\end{abstract}

\section{Introduction}

Diabetes mellitus (abbreviated as diabetes or DM) is a chronic disease manifested by an increased blood sugar level. "Several pathogenic processes are involved in the development of diabetes. These range from autoimmune destruction of the $\beta$-cells of the pancreas with consequent insulin deficiency to abnormalities that result in resistance to insulin action." [2] According to International Classification of Diseases (ICD-10, version 2016), diabetes is included in the "Endocrine, nutritional and metabolic diseases" chapter [3].

There are two forms of diabetes - Type 1 diabetes and Type 2 diabetes. Type 1 is described by the body's loss of the ability to form its own insulin; people affected by this type will be reliant on regular insulin injections for the rest of their life. It affects approximately 5-10\% of all patients [4]. Type 2 diabetes mellitus is considered as a civilization disease that develops in individuals who have a predisposition to it. Obesity, lack of physical activity and stress are generally attributed to unhealthy lifestyle and poor eating habits. It affects the rest of diabetic patients, i.e. 90-95\% [4].

Diabetes is a disease that is not currently curable [5]. The main consequences are chronic complications that reduce the quality of patients' lives. The most serious chronic complications include diabetic retinopathy (visual impairment), diabetic nephropathy (affection or kidney disease), diabetic polyneuropathy (nerve involvement), arteriosclerosis, atherosclerosis resulting in unexpected death, myocardial infarction, lower limb and vascular stroke events [5]. The main goal of current diabetes treatments is to improve the quality and prolong life of patients with this disease [6].

MHealth, defined as "the delivery of healthcare services via mobile communication devices" [7], is an essential part of eHealth [8]. Neelie Kroes, Dutch politician and vice-president of the European Commission for Digital Agenda, said: „Evidence shows that eHealth pays off in terms of efficiency gains, health and welfare improvements and increased care worker productivity. " [9]. mHealth focuses on prevention and helps increase the efficiency of the healthcare system. New challenges and many budget 
constraints are emerging as Western world population is aging. Based on previous studies [10], [11], [12], mHealth could be one of the tools to solve these problems. mHealth can help diagnose chronic illnesses earlier and is therefore already at the onset of the disease, which allows for a timely start of the treatment of diabetes.

The main aim of this paper is to specify mHealth implementation into diabetes type 2 diagnostic and treatment processes.

The following research methods have been used: firstly, type 2 diabetes patient care was analysed based on the case study in Czech healthcare facilities; second, mHealth devices and applications were identified and implemented into the patient care processes. The structure of this paper corresponds to the above.

\section{Type 2 diabetes patient care}

\subsection{Diabetes diagnosis and initial interview}

In most cases, patients with type 2 diabetes have no symptoms, they are asymptomatic. Very often, higher blood sugar level is identified accidentally when blood is taken in connection with another disease or medical procedure. In most cases, patients do not take higher blood glucose level seriously, defending it, for example, by saying they ate too many sweets the day before. However, if the withdrawal takes place on the next day, then a healthy person will have higher blood sugar level and the blood glucose level will be correct.

When the patient is diagnosed with type 2 diabetes, a practitioner initiates a complex therapy involving diet and regimen according to the patient's age and overall condition. Once pharmacological treatment has started, the first choice is metformin, unless the patient has contraindication to this treatment.

If a practitioner assesses that a patient's condition requires specific treatment (such as with insulin or a combination of oral antidiabetic agents), patients are referred to a specialist - a diabetologist.

\subsection{Examination}

Since the lack of discipline, not every patient will visit the diabetologist immediately. When a patient decides to undergo the examination (whether due to a complication, fear for his or her health, or urging by a practitioner or family), the examination consists of several steps: The first step is the collection of anamnestic data, when the diabetologist asks the patient for his/her current and pre-existing medical condition, for symptoms of illness, risk factors, lifestyle, and so on. Physical and laboratory examinations should be also included.

Health records are classified as sensitive; therefore, healthcare facilities and their employees must follow a specific law on the protection of personal data. Doctors can process sensitive patient data, as the law authorizes data processing in the provision of health services and health insurance. Information about the health condition of the patient, such as the provider's identification data, patient's identification data, gender, nurse's name, etc., must be included in the health records. The documentation may include a final diagnosis, suggestion of a possible solution to the patient's medical condition, information on the possible course of treatment, current health status, vaccinations, medical reports, or a record of recognition or termination of the patient's incapacity for work etc. The patient's name and date of birth are kept and healthcare facilities are responsible for recording all of them. If the healthcare records are electronically documented, each record must contain an electronic signature and all of them must be archived.

\subsection{Diagnostics}

Patients are diagnosed based on the results of previous examination phase - laboratory tests. In our case study, type 2 diabetes diagnosis is positive for the patients when:

(1) a randomized plasma glucose assay is greater than or equal to $11.1 \mathrm{mmol} / 1$ in combination with a clinical symptom;

(2) plasma glucose concentrations are greater than or equal to $7.0 \mathrm{mmol} / 1$ (on an empty stomach); 
(3) glucose plasma concentration at oGTT in $120 \mathrm{~min}$. is greater than or equal to $11.1 \mathrm{mmol} / 1$.

The process of the first visit to the diabetologist including phases B. Examination and C. Diagnostics is illustrated by Fig. 1.

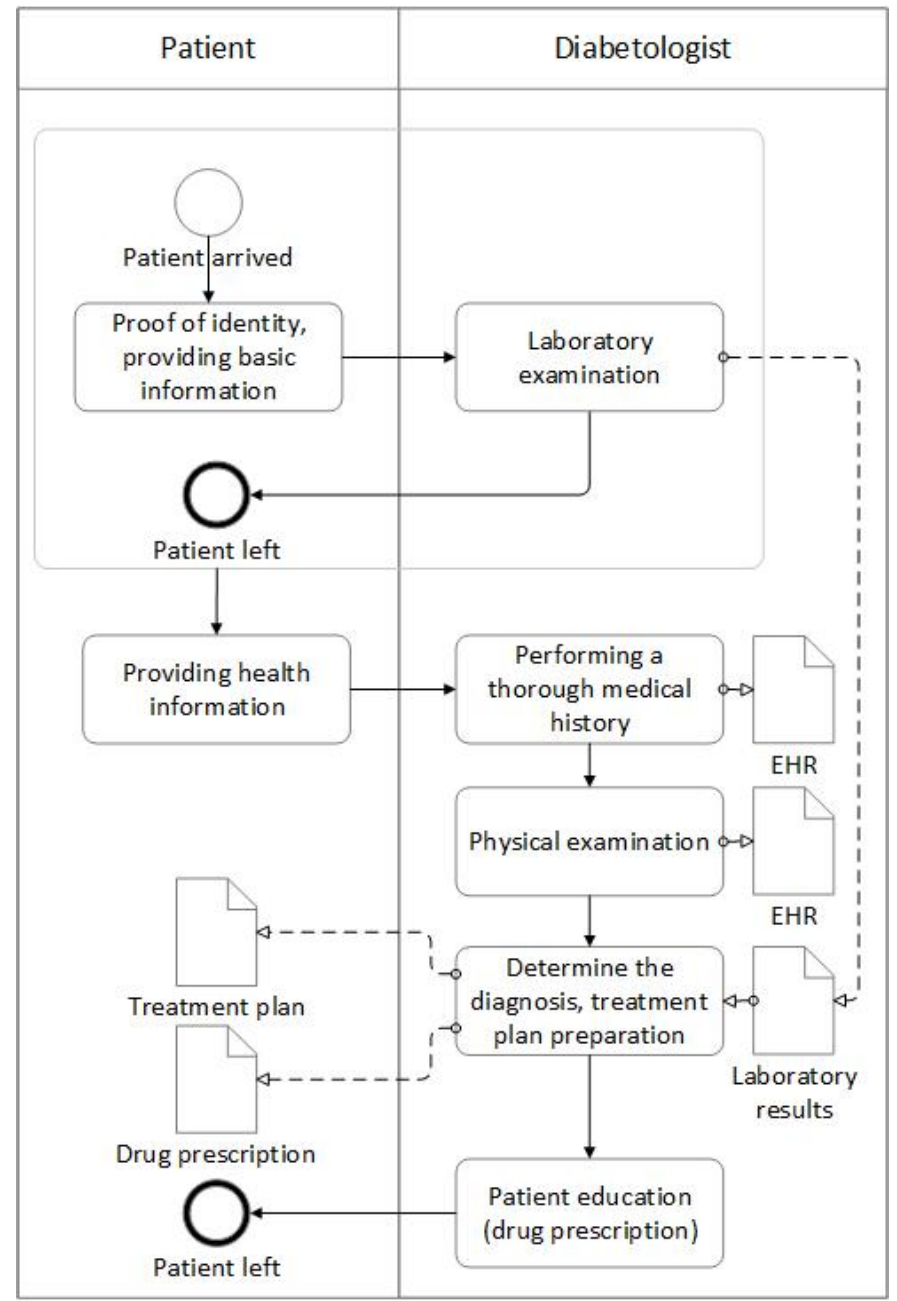

Fig. 1. Visit to diabetologist, examination and diagnosis

\subsection{Therapy}

When the diagnosis is positive, the patient is given a treatment plan, which is started according to patient's condition. In any case, diet, healthier lifestyle and higher physical activity is recommended to the patient. Usually, metformin will be implemented immediately. Based on our case study, no patient with type 2 diabetes should go without metformin. Although this is not fully respected, the number of such patients is minimal. Other drugs are used for patients who are intolerant to metformin, possibly combined, often with insulin.

The provision of health services must be in accordance with the law (of the country) which defines healthcare, patient and healthcare facilities. The law determines the competence to practice healthcare profession and the authorization to provide healthcare services. It also deals with patient rights (providing patient-assisted help) and duties (adherence to treatment, order management, etc.) during the therapy.

\subsection{Regular check-ups}

Individual check-ups are based on the patient's medical history, physical and laboratory examination. Intervals between check-ups are individual. If the patient is treated with non-pharmacological treatment (including diet, healthy lifestyle, and higher levels of physical activity), the check-ups will be scheduled 
1-2 times per year. In the case of pharmacological treatment, 1 check-up will be scheduled every 3-6 months. If the patient uses insulin in his or her treatment, 1 check-up will be scheduled every 2-3 months. In the beginning, especially for the patients who are re-administering insulin, check-ups can be performed several times a week. Remote consultations with a diabetologist through a mobile phone - about once a week to adjust the dose of insulin - are possible. The process of regular check-ups is illustrated by Fig. 2.

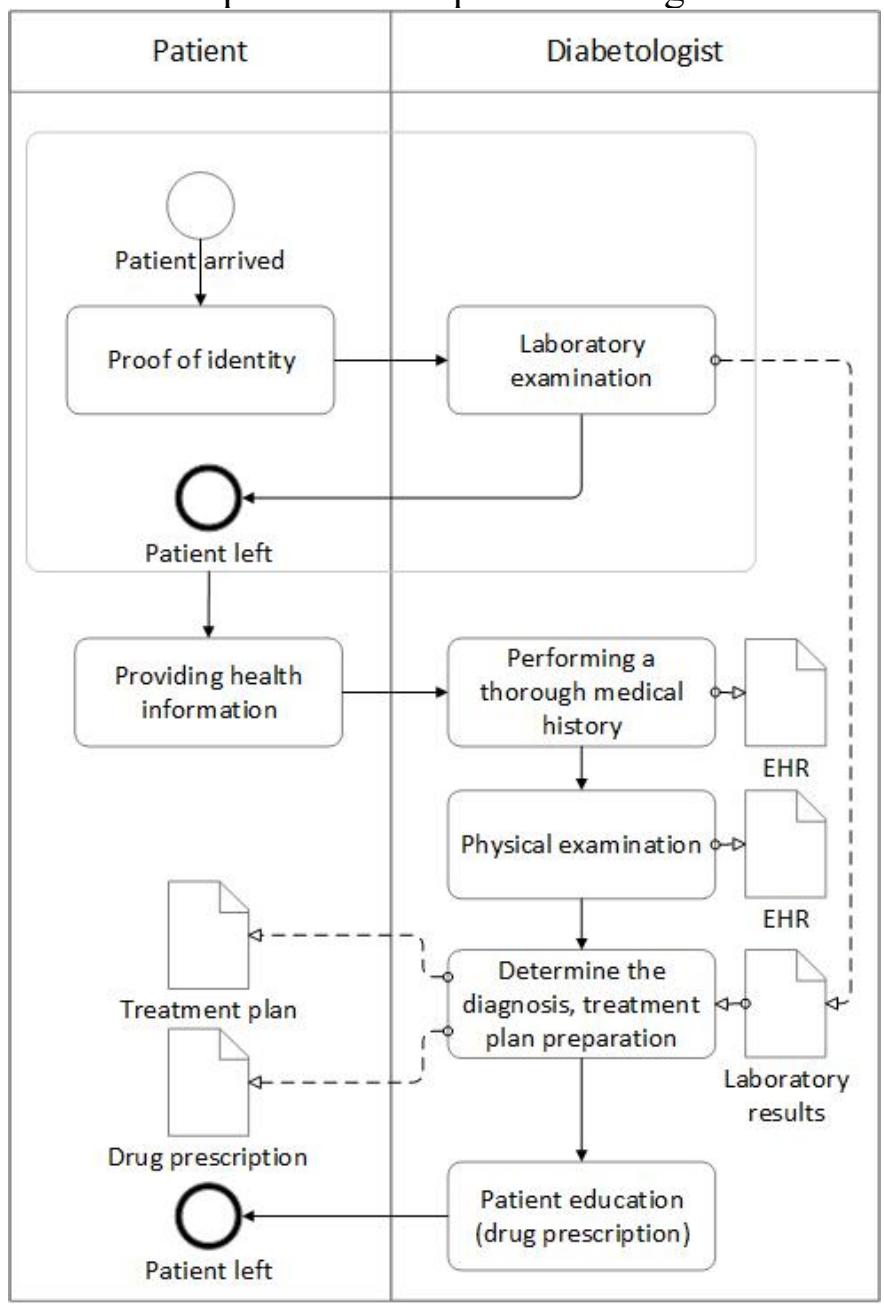

Fig. 2. Regular check-ups

\section{Type 2 diabetes treatment using mHealth}

Based on a market survey we concluded that smart devices such as glucometers, activity monitors and scales are available and ready to be implemented into the process. The examples of smart glucometers with Bluetooth technology or the ability to send data to a mobile phone are iHealth GLUCO BG 5, iHealth ALIGN BG1 and Diamond MINI FC Glucometer from eVito.

Currently, the App Store offers approximately 1,250,000 applications, which are divided into 23 categories. The "Medical" and "Health and Fitness" categories include applications related to health and healthy lifestyle. ${ }^{[13]}$ Google Play offers almost the same number of applications as App Store. 1,400,000 applications are divided into several categories including "Medical" and "Health and Fitness". [14]

For our case study, we supplied the patient with a smart glucometer, pressure gauge, pedometer, pulse oximeter and scale.

The first visit to the doctor and the examination consists of the same procedure as if it was carried out without the implementation of mHealth, the difference being that the doctor recommends the patient to 
implement monitoring devices with the necessary technology (Bluetooth or WiFi) to the treatment plan see Fig. 3.

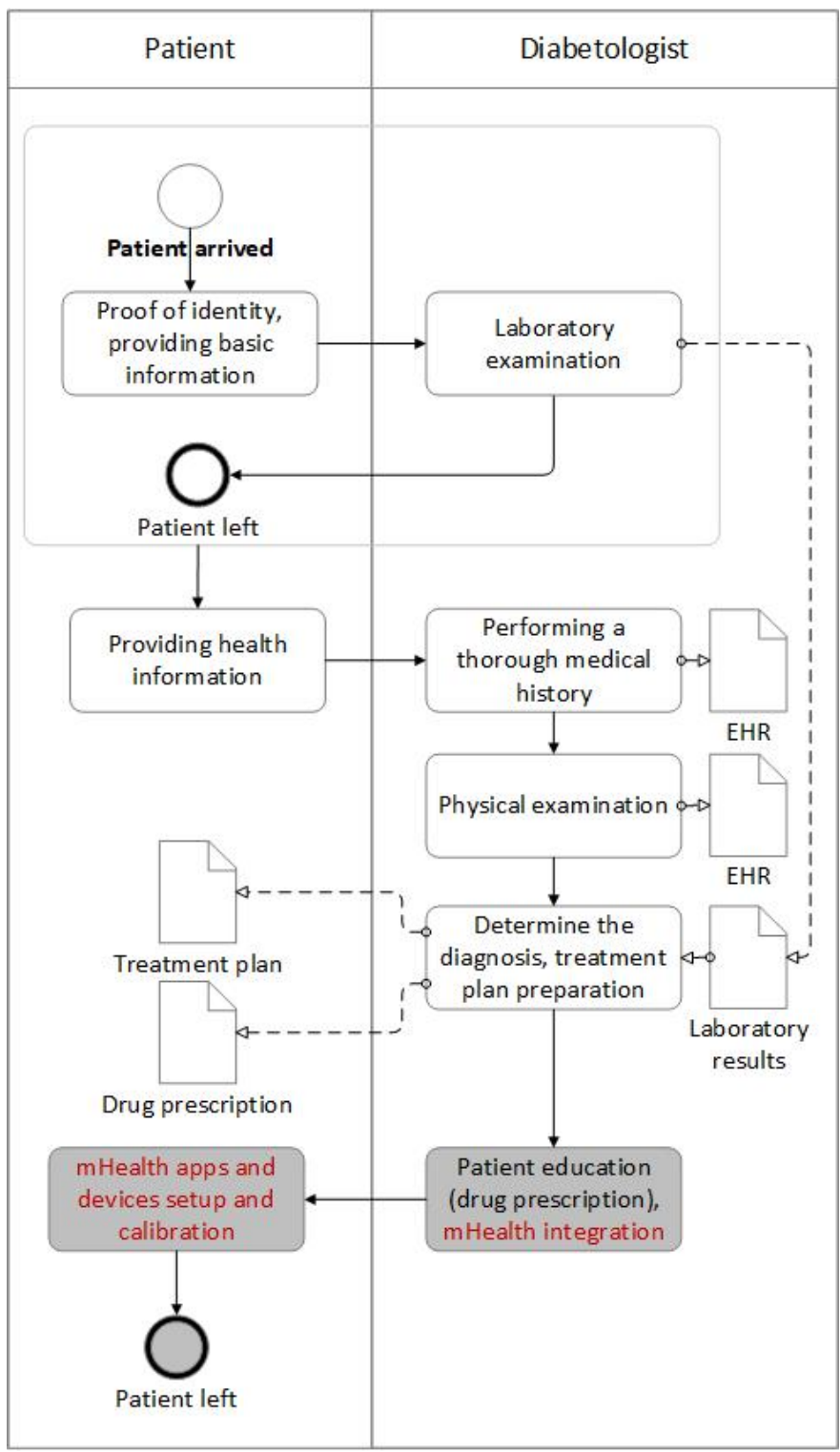

Fig. 3. Visit to diabetologist, examination and diagnosis using mHealth

The prerequisite is that the patient owns a smartphone and is interested in the test. The difference of patients with type 2 diabetes is their age distribution. According to our case study, most of the patients suffering from type 2 diabetes do not use modern technology and the use of such applications would be challenging and not practical due to their age. For some patients, even measuring with a conventional glucometer and applying the right dose of insulin (or other medication) is a complex operation. With the passing of time, with new modern technologies and the aging of the current generation which uses modern technology, smartphones and applications in their day-to-day lives without problems, it will become easier and more natural to use mobile technology to measure and monitor diabetes. At the same time, it would be advisable for manufacturers to continue to focus on improving quality, optimizing, and making applications more user-friendly, making it easier and more user-friendly. 


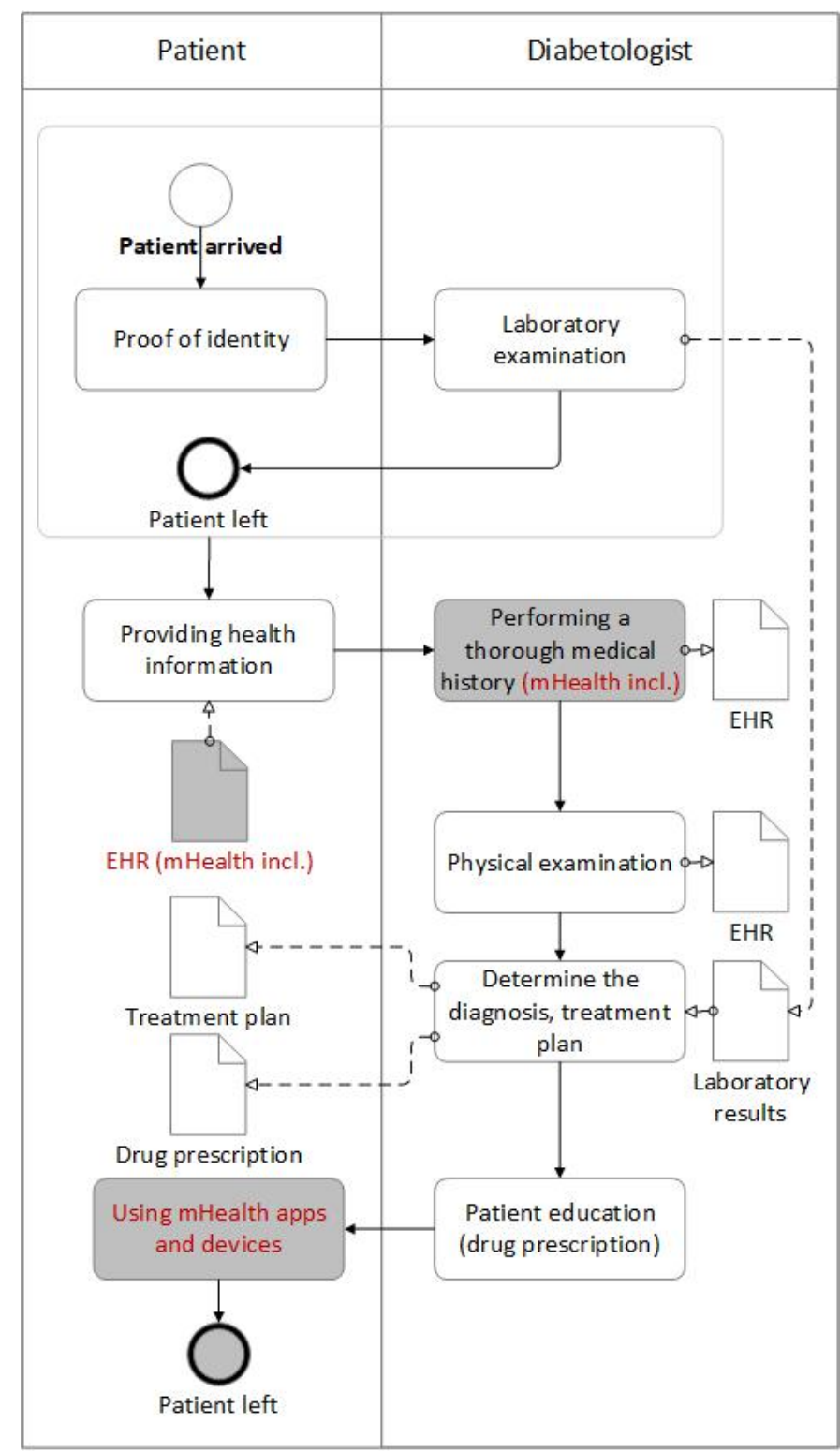

Fig. 4. Regular check-ups using mHealth

If the patient chooses to purchase mHealth equipment, it is important to pair the device properly and calibrate it with his or her mobile phone. Then, the treatment process will be shortened and the diabetic patient consults the measured values - according to blood glucose level, physical activity, blood pressure or measured weight. Physical and laboratory testing is still part of the check-up, see Fig. 4.

After receiving results, the diabetologist evaluates the success of the treatment plan and determines following treatment course. The patient is once again educated about the illness, his or her life, and the treatment plan is implemented.

\section{Assessment of mHealth implementation and its benefits}

After 10 days of testing and measurement, we have done an interview to evaluate the effects of mHealth implementations. The interviews were done with both patients and doctors. The most important results are stated below. 


\subsection{Patient}

It makes life easier especially because patients do not have to manually write down any results. All measurements via smart devices are automatically transferred. The measured values are stored both in the app itself and in the Healthy Apps app that is installed. Patients have a very good overview of their health situation and do not have to worry about possible unexpected complications. Regular check-ups are a bit faster for the physician due to the mobile health service, the diabetologist has a better overview of the patient's condition after displaying the measured values, he or she does not have to ask (the history is still part of the examination) and rather assesses the measured values.

\subsection{Doctor}

By using mobile health features, the work of a diabetologist will be easier, but on the other hand, there might be some extra work and additional processes.

Regular check-ups do not need to be so frequent for a well-compensated patient, the examination itself takes less time - the diabetologist should have a better understanding of the patient's health during the past period.

Additional work for diabetologists consists of monitoring individual patients (using online solutions, using data sharing through cloud computing and web interface) and their measured values. In case of changes in blood glucose level or deterioration, the physician should contact the patient via telephone, SMS, e-mail or arrange for an earlier check-up with the patient.

Patients' regular monitoring by the diabetologist could prevent more serious problems as the change in the patient's medical condition would begin to be resolved at the outset. It often happens that the patient does not feel well, knows that he or she has a health problem, but does not contact the doctors because of fear (check with a doctor, diagnosis). If the patient was under remote supervision, the doctor would call the patient by him or herself and consult the problem.

\section{Conclusion}

The aim of this paper is to specify mHealth implementation into diabetes type 2 diagnostic and treatment processes. The results of the first part of our analysis have shown the opportunities of implementing mHealth into diabetes type 2 diagnosis and treatment. The final model of these processes brings the mHealth implementation and usage which was also assessed by both patients and doctors.

In a timeframe of approximately four years (according to the eHealth Action Plan 2012-2020) [15], the healthcare system should be much more interconnected (not only between the patient and the doctor but also among institutions and medical facilities) and almost everything should be done on electronic basis. Diabetologists should have access to at least basic health data about their patients from other healthcare facilities (general practitioner, hospital examination). Diabetologist should already have an overview of the problems the patient suffers from and the approximate state of their health.

In the context of ongoing screening, patients will continually share their measured values with their diabetologist who will be aware of the health status of their patients. Anylaboratory testing is important for each check-up. Laboratory measurements and results will still be available only in specialized laboratories, but the patients will not have to visit their diabetologist, they will visit the nearest certified laboratory facility. However, the diabetologist's interpretation of the results will still be important. Values will have to be assessed not only regarding common standards but also with respect to the particular patient, his or her past results and associated diagnoses. In the case of necessary medicines, an electronic prescription might be used.

In theory, patients with a good health status and good compensation of diabetes are not necessary to seeing a doctor for regular check-ups. The patient should visit the diabetologist once a year during a complete examination.

The applicability of our results is twofold. First, healthcare facilities can use the results when implementing mHealth into the processes; the results can also be used by IS/ICT professionals when designing new mHealth (eHealth) elements. Second, the results can be used in further research. It is 
necessary to expand the case study by seeking factors influencing the implementation (motivation of patients and doctors, health insurance, etc.)

\section{Acknowledgment}

This paper was written thanks to the long-term institutional support of research activities by the Faculty of Informatics and Statistics, University of Economics, Prague.

The support of the doctors and managers at all participating healthcare facilities is gratefully acknowledged.

\section{References}

[1] WHO. "World Health Statistics 2016: Monitoring Health for the SDGs Sustainable Development Goals". World Health Organization, 2016. ISBN 9241565268.

[2] ADA. "Diagnosis and Classification of Diabetes Mellitus". Diabetes Care [online]. January 2010. Vol. 33, no. Suppl 1, p. S62-S69. DOI 10.2337/dc10-S062. Available from: http://www.ncbi.nlm.nih.gov/pmc/articles/PMC2797383/.

[3] WHO. "ICD-10 Version:2016". [online]. 2016. [Accessed 19 August 2017]. Available from: http://apps.who.int/classifications/icd10/browse/2016/en.

[4] WEBMD. "Diabetes: Differences Between Type 1 and 2-Topic Overview". [online]. 2017. [Accessed 24 August 2017]. Available from: http://www.webmd.com/diabetes/tc/diabetesdifferences-between-type-1-and-2-topic-overview.

[5] WHO. "WHO | Diabetes". WHO [online]. 2017. [Accessed 24 August 2017]. Available from: http://www.who.int/mediacentre/factsheets/fs312/en/.

[6] CDS. "Úvod | Aktivity | Národní diabetologický program | Národní diabetologický program 2012 2022”. Česká diabetologická společnost. [online]. 2012. [Accessed 24 August 2017]. Available from: http://www.diab.cz/narodni-diabetologicky-program-2012-2022\#3.

[7] TORGAN, Carol. “The mHealth Summit: Local \&amp; Global Converge - Kinetics". [online]. 2009. [Accessed 21 August 2017]. Available from: http://caroltorgan.com/mhealth-summit/.

[8] NIH. "PAR-14-028: Mobile Health: Technology and Outcomes in Low and Middle Income Countries (R21)". [online]. 2015. [Accessed 6 August 2015]. Available from: http:/grants.nih.gov/grants/guide/pa-files/PAR-14-028.html.

[9] EUROPEAN COMMISSION. "European Commission - PRESS RELEASES - Press release Digital Agenda: Kroes and Dalli welcome Council Presidency eHealth Declaration on delivering better health care". [online]. 2011. [Accessed 19 August 2017]. Available from: http://europa.eu/rapid/press-release_MEMO-11-287_en.htm.

[10] WHO. mHealth: "New horizons for health through mobile technologies. Observatory". [online]. 2011. Vol. 3, no. June, p. 66-71. DOI 10.4258/hir.2012.18.3.231. Available from: http://www.webcitation.org/63mBxLED9.

[11] MCKINSEY \& COMPANY and GSMA. "mHealth: A new vision for healthcare". Mckinsey \& Company. 2010. P. 1-20. DOI 10.1186/1472-6963-14-S2-P87.

[12] HILLESTAD, Richard, BIGELOW, James, BOWER, Anthony, GIROSI, Federico, MEILI, Robin, SCOVILLE, Richard and TAYLOR, Roger. "Can electronic medical record systems transform health care? Potential health benefits, savings, and costs". Health affairs. 2005. Vol. 24, no. 5, p. 1103-1117.

[13] APPLE. “App Store Downloads on iTunes". [online]. 2015. [Accessed 6 August 2015]. Available from: https://itunes.apple.com/us/genre/ios/id36?mt=8.

[14] XU, Wenlong and LIU, Yin. "mHealthApps: A Repository and Database of Mobile Health Apps". JMIR mHealth and uHealth [online]. 18 January 2015. Vol. 3, no. 1, p. e28. 
[Accessed 5 August 2015]. DOI 10.2196/mhealth.4026. Available from: http://mhealth.jmir.org/2015/1/e28/.

[15] EUROPEAN COMMISSION. "eHealth Action Plan 2012-2020 - Innovative healthcare for the 21st century". 2012. 